\title{
Comparison of the Effects of Aprepitant and Ondansetron Individually and Combining on Postoperative Nausea and Vomiting after Laparoscopic Cholecystectomy
}

\author{
Farhang Safarnejad ${ }^{1}$, Karim Nasseri ${ }^{2}$, Reza Karami ${ }^{3}$
}

\begin{abstract}
Background: Nausea and vomiting are one of the most common postoperative complications that cause unpleasant feelings and delays in the discharge of patients. This study aimed to compare the effect of aprepitant, ondansetron, and their combination on the severity of nausea and vomiting after this procedure for finding a safe and less indisposition regimen.

Materials and methods: This study was performed on patients aged 18-50 who had been diagnosed with symptomatic cholelithiasis and who underwent laparoscopic cholecystectomy under general anesthesia. This study was done single-blinded. Patients were categorized into three groups (the recipient of aprepitant, the recipient of ondansetron, and the group receiving ondansetron and aprepitant simultaneously) and the rate of nausea and vomiting was measured at 6 and 24 hours after the operation.

Results: The results of one-way analysis of variance analysis and Kruskal-Wallis showed that there was a significant difference between the treatment groups regarding the severity of nausea and vomiting after surgery $(p<0.001)$. The severity of nausea and vomiting in the group receiving ondansetron plus aprepitant is less than the other two groups.

Conclusion: A combination of ondansetron plus aprepitant can reduce nausea and vomiting after surgery while the effect of aprepitant is much more than ondansetron.

Keywords: Aprepitant, Cholecystectomy, Ondansetron, Vomiting.

World Journal of Laparoscopic Surgery (2021): 10.5005/jp-journals-10033-1440
\end{abstract}

\section{INTRODUCTION}

Nausea and vomiting are common serious postoperative complications that cause unpleasant feelings and hospital stay elongation. ${ }^{1}$ The annual cost of postoperative nausea and vomiting has been reported in the United States for several hundred million dollars. ${ }^{2}$ Postoperative nausea and vomiting in $20-30 \%$ of patients and are the second most common postoperative complications. ${ }^{3}$ Its incidence is $37-90 \%$ without prophylaxis. ${ }^{4}$ Nausea and vomiting may lead to serious but rare complications of aspiration and postoperative hypoxemia, fluid and electrolyte disorders, and dehiscence of the surgical site. 'Some factors, such as age, sex, previous history of nausea and vomiting, motion sickness, type of surgery, duration of anesthesia and surgery, and anxiety of the patient and parents are the factors influencing nausea and vomiting, which cannot be controlled by the anesthesiologist. ${ }^{5}$ Some studies demonstrated that type of operation may be associated with postoperative nausea and vomiting, but there are controversies in this regard. ${ }^{1}$

An increase in surgery and anesthesia duration leads to an increase in the risk of nausea and vomiting, which is probably due to the accumulation of anesthetizing agents. The incidence of nausea and vomiting rises from $2.8 \%$ in patients with a duration of less than 30 minutes to $27 \%$ in patients with an operation duration between 151 minutes and 180 minutes. The duration of anesthesia increases the risk of nausea and vomiting up to $59 \%$ per 30 minutes. ${ }^{6}$ Of course, some of the factors affecting the development of postoperative vomiting and vomiting are under the control of anesthesiologists who need to pay attention to controlling this complication, including
${ }^{1}$ Department of Surgery, School of Medicine, Kurdistan University of Medical Sciences, Sanandaj, Kurdistan Province, Iran

${ }^{2}$ Department of Anesthesiology, Faculty of Medicine, Kurdistan University of Medical Sciences, Sanandaj, Kurdistan Province, Iran

${ }^{3}$ Department of Minimal Invasive Surgery, School of Medicine, Iran University of Medical Sciences, Tehran, Iran

Corresponding Author: Reza Karami, Department of Minimal Invasive Surgery, School of Medicine, Iran University of Medical Sciences, Tehran, Iran, Phone: +98 9133415767, e-mail: reza_karami1367@yahoo.com

How to cite this article: Safarnejad F, Nasseri K, Karami R. Comparison of the Effects of Aprepitant and Ondansetron Individually and Combining on Postoperative Nausea and Vomiting after Laparoscopic Cholecystectomy. World J Lap Surg 2021; 14(1):39-42.

Source of support: This study was funded by Kurdistan University of medical sciences

Conflict of interest: None

premedication, type of anesthesia, anesthetic drugs during surgery, postoperative management, and anti-anesthetic drugs. It has been reported that patients who receive general anesthesia are 11 times more likely to have nausea and vomiting after surgery than those who receive regional or block anesthesia for the treatment of chronic pain. The use of intubation due to stimulation of pharyngeal mechanoreceptors is believed to be associated with an increased risk of postoperative nausea and vomiting. ${ }^{6}$ Choosing the right treatment and appropriate intervention in controlling nausea

() Jaypee Brothers Medical Publishers. 2021 Open Access This article is distributed under the terms of the Creative Commons Attribution 4.0 International License (https://creativecommons.org/licenses/by-nc/4.0/), which permits unrestricted use, distribution, and non-commercial reproduction in any medium, provided you give appropriate credit to the original author(s) and the source, provide a link to the Creative Commons license, and indicate if changes were made. The Creative Commons Public Domain Dedication waiver (http://creativecommons.org/publicdomain/zero/1.0/) applies to the data made available in this article, unless otherwise stated. 
and vomiting can improve patient satisfaction with medical and anesthetic services and promote the level of activities and the patient's faster recovery. ${ }^{6}$

Different factors in the incidence of post-surgical nausea and vomiting play a role in spinal anesthesia, the most important factor being the increased stimulation of vagus due to sympathetic inhibition. Other factors, such as hypotension (systolic blood pressure less than $80 \mathrm{~mm} \mathrm{Hg}$ ), block-level higher than thoracic fifth space, adding substances such as vasoconstrictors, neostigmine, and opioids to the anesthetic, increase the chance of nausea and vomiting. Reduced blood pressure causes brain stem cell ischemia, which leads to stimulation of the vomiting center in the brain stem. Also, hypotension is associated with ischemia of the intestines and the release of nausea-like substances, such as serotonin. ${ }^{2}$

Several methods and drugs, including metoclopramide, droperidol, a specific 5-HT antagonist, propofol, and dexamethasone, are used to treat this condition. The most commonly used drug is metoclopramide, which is characterized by the risk of extrapyramidal symptoms and complications, such as drowsiness, dizziness, and headache. ${ }^{7}$ Therefore, proper treatment achievement is one of the concerns in this regard, so that research in reducing postoperative nausea and vomiting focuses on low-price methods and drugs. The drug should have the greatest effect and duration and the least complication. ${ }^{6}$

The aprepitant is a long-acting agonist of neurokinin-1 (NK1), whose half-life is 9-12 hours. ${ }^{8}$ The neurokinin receptor 1 is known to be nausea-like as a receptor with high effect in both acute and chronic forms. ${ }^{9}$ This drug has been approved by FDA for nausea prophylaxis induced by chemotherapy. This oral medication is known as an effective drug for opioid nausea. On the other hand, it has no sedative effect and its use in obese and apnea patients is quite safe during surgery and anesthesia. ${ }^{10}$

The other drugs include serotonin antagonists, such as ondansetron and dolasetron, which have a good effect on nausea and vomiting. ${ }^{10}$ Ondansetron complications are headache, stomach upset, dizziness, flushing of the injection site, and arrhythmia. ${ }^{11}$

Another risk factors for postoperative nausea and vomiting are laparoscopic. ${ }^{12}$ Laparoscopy is used to diagnose and treat many diseases. Pneumoperitoneum, during laparoscopy, can stimulate the vagus nerve and increase the chance of nausea and vomiting. Postoperative nausea and vomiting cause, discharge delay, dehydration, wound dehiscence, pulmonary aspiration, patient dissatisfaction, and increased costs. ${ }^{13}$ Regarding the high prevalence of laparoscopic cholecystectomy and postoperative nausea and vomiting, in our study, we compared the effect of aprepitant, ondansetron, and combination of ondansetron and aprepitant, on postoperative nausea and vomiting, to introduce a regimen that is safe and low cost.

\section{Materials and Methods}

After approving the plan and performing the necessary coordination, the study was performed as a double-blind clinical trial after receiving written consent from patients. A total of 90 patients which were candidate for laparoscopic cholecystectomy under general anesthesia were admitted to the hospital. Inclusion criteria for the study include all patients are women aged 18-50 years, and the American Association of Anesthesiologists has a health level of 1 and 2, and at least two APFEL criteria (including female gender, non-smoker, having a history of PONV, and use of $100 \mathrm{mcg}$ fentanyl or equivalent). Exclusion criteria include a woman aged over 50 and under 18, patients with a health status of 3,4,5 the American Association of Anesthesiologists, patients with regional anesthesia cholecystectomy, patients with any systemic disease, such as diabetes, asthma, cardiovascular disease, gastroesophageal reflux, severe obesity, pregnancy, lactation, liver and kidney diseases, neuromuscular diseases, psychiatric disorders, alcohol addicts and drug and smoker, and acute cholecystitis.

This study is double-blind. For the blinding of drugs and placebo, they are packaged by a pharmacist in unnamed packages and encoded and packaged into the operating room. After completing the design and measuring the indicators before the patient data analysis, the codes are delivered from the pharmacist and the case and control groups are identified.

After insertion of the patient into the operating room, venous access of the patient had the fluid infusion, and crystalloid infusion (Ringer's serum) started at $500 \mathrm{cc}$ and vital signs were monitored. General anesthesia was induced and patients were divided into three groups: at the same time as an anti-nausea drug, ondansetron $4 \mathrm{mg}$ (Tehran Chemistry Company) was administered intravenously and placebo capsule an hour before the operation. In another group, an 80-mg aprepitant capsule from Tehran Chemistry Company received an hour before the operation with placebo ampulla during operation and in the third group, an 80-mg capsule of aprepitant 1 hour before the operation and $4 \mathrm{mg}$ ondansetron was given during the operation.

The patient was monitored for at least 30 minutes in the recovery room and then was delivered to the surgical ward. The severity of nausea and vomiting of patients at 6 and 24 hours after the operation as early and late symptoms using visual analog scale (VAS) criteria $^{14}$ was recorded in the questionnaire. Nausea and vomiting were measured based on VAS criteria. A $100 \mathrm{~mm}$ graduated line, the first of which without nausea, and the end of it is unbearable nausea. The occurrence of nausea and vomiting is collected through a patient's inquiry. The need for antiemetic medication is questioned by the nurse. Patient information is categorized in separate tables and analyzed by SPSS software.

\section{Results}

The mean age of the patients was 39.14 with a standard deviation of 7.78 years (Fig. 1). The highest age in this study was 50 years and the lowest 21 years. The mean severity of nausea and vomiting at

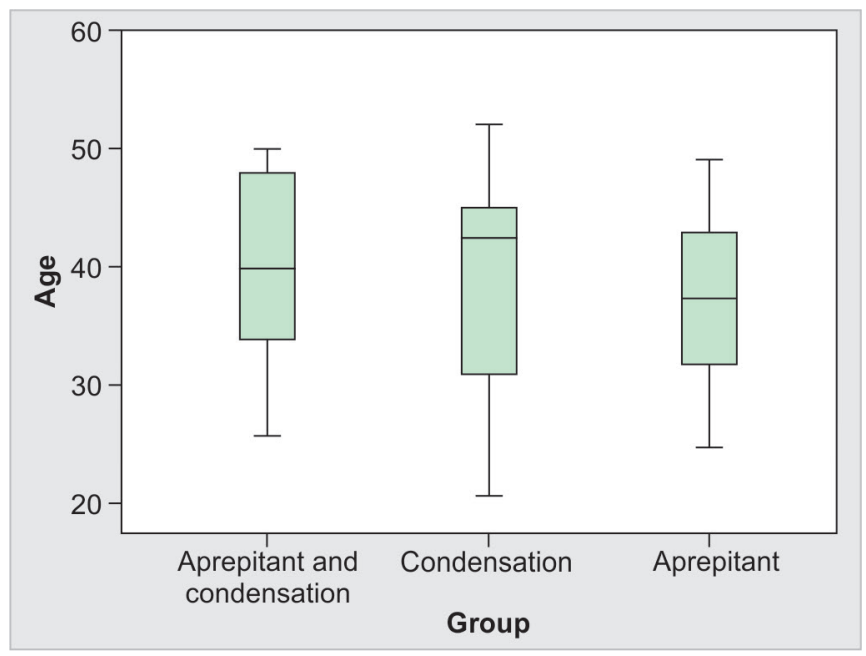

Fig. 1: Box plot of age distribution in groups 


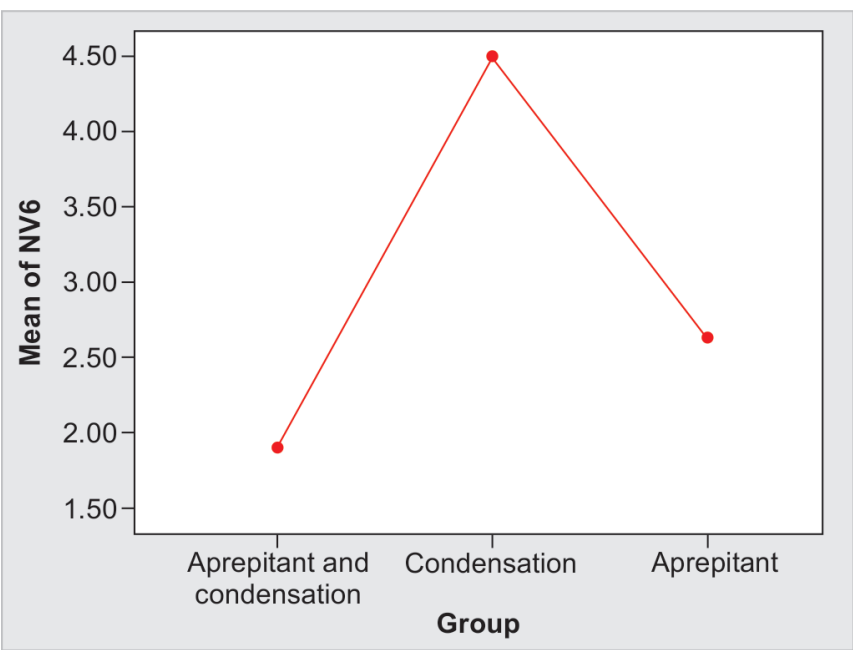

Fig. 2: Means plot of nausea and vomiting at 6 hours in groups

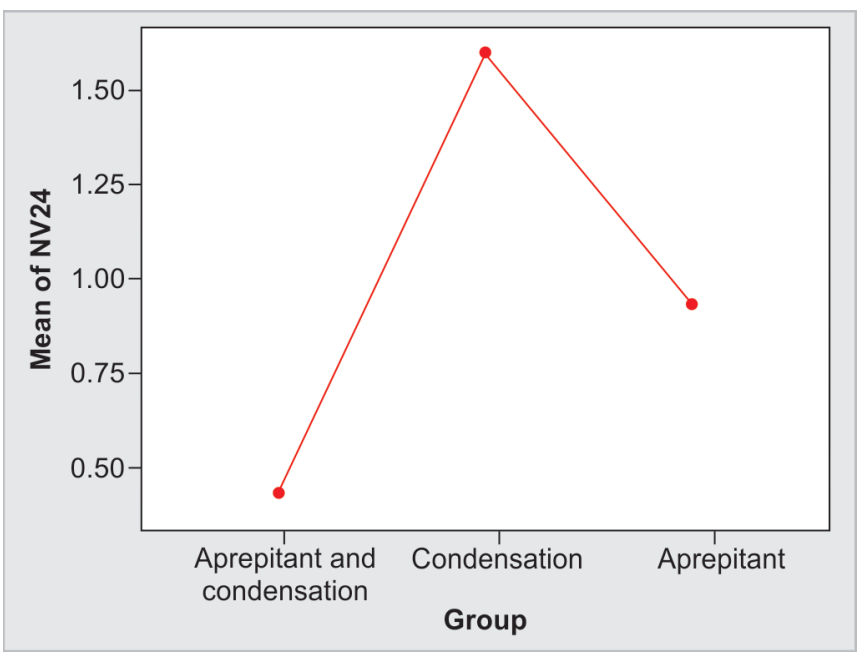

Fig. 3: Means plot of nausea and vomiting at 24 hours in groups

6 hours after the operation was 3.01 with a standard deviation of 1.71 (Fig. 2). The highest severity of nausea and vomiting was in subjects equal to 7 and lowest of zero. The mean severity of nausea and vomiting during the 24 hours after the operation was 0.99 with a standard deviation of 0.98 (Fig. 3). The highest severity of nausea and vomiting was in patients equal to 3 and the lowest was zero.

Considering that the $p$-value of the one-way analysis of variance is more than 0.05 , it can be concluded that there is no significant difference between the studied groups in terms of the mean age, so the groups are conformed.

Considering that the amount of results from one-way analysis of variance is less than 0.05 , it can be concluded that there is a significant difference between the groups in terms of severity of nausea and vomiting at 6 hours.

Considering the results of Tukey follow-up, it can be concluded that there is a significant difference between the treatment group receiving ondansetron and the two other groups, thus the severity of nausea and vomiting in the group receiving ondansetron is more than the other two groups.

Considering the result obtained from Kruskal-Wallis is less than 0.05 , it can be concluded that there is a significant difference between the groups in terms of severity of nausea and vomiting in 24 hours. According to the calculated mean for nausea and vomiting severity in the groups, it is concluded that the highest severity of nausea and vomiting has occurred in the ondansetron group and the lowest in the group receiving the aprepitant plus ondansetron.

On the other hand, according to Pearson's correlation test, there was no significant difference between age and nausea and vomiting.

\section{Discussion}

Nausea and vomiting are common complications in the postoperative period, which causes bad and unpleasant feelings and delays in discharge of patients. Nausea and vomiting may lead to serious but rare complications, such as aspiration and postoperative hypoxemia, fluid and electrolyte imbalance, and the dehiscence of the surgical site. Postoperative nausea and vomiting in $20-30 \%$ of patients and are the second most common postoperative complication. Some factors, such as age, sex, previous history of nausea and vomiting, motion sickness, type of surgery, duration of anesthesia and surgery, and anxiety of the patient and parents are among the factors influencing nausea and vomiting that are not under the control of anesthesiologist. ${ }^{14}$ Several methods and drugs, including metoclopramide, droperidol, a specific HT-5 antagonist, propofol, and dexamethasone, are used to treat this condition. The most commonly used drug is metoclopramide, which has the potential for extreme opiates and side effects, such as drowsiness, dizziness and headache, and low effect duration. Research in reducing postoperative nausea and vomiting focuses on effective low-dose medications and therapies. Medication should have the greatest effect and least complication. ${ }^{15}$ This study aimed to compare the effects of aprepitant and ondansetron separately and their combination on postoperative nausea and vomiting after laparoscopic cholecystectomy. The mean age of patients in the study was $39.14 \pm 7.78$ years, which was $42.17 \pm 14.03$ in similar studies. ${ }^{15}$ The patients were conformed in different age groups, which were similar in parallel studies. ${ }^{14,15}$

Comparisons between groups were performed using statistical tests. According to the results, the severity of postoperative nausea and vomiting at 6 and 24 hours after surgery showed that in the group which administered ondansetron and aprepitant simultaneously, the severity of nausea and vomiting is less than the other two groups. The existence of two different mechanisms of action for both ondansetron and aprepitant drugs can lead to the conclusion that co-administration of both drugs improves the outcome and reduces the incidence of vomiting in patients.

Comparing nausea and vomiting in other groups based on statistical tests, it was demonstrated that the level of nausea and vomiting at 6 and 24 hours after surgery was significantly different between the groups of aprepitant and ondansetron, this difference in mechanism leads to different effects of these two drugs, indicating that the blocking mechanism of the neurokinin receptor 1 has more effect on vomiting than the serotonin 5-HT3 receptor block pathway. On the other hand, there is not a significant difference in the rate of nausea and vomiting between the groups of aprepitant and the simultaneous aprepitant and ondansetron group, which indicates the potent antiemetic effect of the aprepitant.

As it was demonstrated, there is no significant relationship between age and severity of nausea and vomiting in different groups. Therefore, based on this study, it can be concluded that age does not have a clear effect on nausea and vomiting after surgery. 
Other findings of the study indicate that there is a significant correlation between nausea and vomiting in 6 and 24 hours after surgery in the aprepitant group and the aprepitant and ondansetron group simultaneously. This result shows the ability to predict the severity of nausea and vomiting 24 hours after surgery based on the severity of nausea and vomiting 6 hours after surgery. In the event of severe symptoms at 6 hours, the symptoms may be more severe at 24 hours. But in general, the severity of symptoms at 24 hours is less than 6 hours.

In a study by Se-Jin Lee et al., they evaluated the effect of aprepitant in preventing postoperative nausea and vomiting in patients undergoing surgery. They demonstrated a significant difference between the aprepitant and the control group at 6 and 24 hours, while the incidence of nausea and vomiting in the aprepitant group was less than the control group. At 6-24 hours, the incidence was less than the first 6 hours, ${ }^{14}$ which is consistent with our study.

Diemunsch et al., in their study, compared aprepitant with ondansetron for the prevention of postoperative nausea and vomiting in major abdominal surgery. They indicated that aprepitant is more effective than ondansetron in reducing nausea incidence on the first day following surgery, which is consistent with our study. ${ }^{16}$

Another study by Vallejo et al. assessed aprepitant in reducing postoperative nausea for 48 hours in patients undergoing plastic surgery. The results demonstrated the severity of nausea was significantly higher ondansetron group comparing with the ondansetron plus aprepitant group, which is in the same way with our study. ${ }^{17}$

\section{Conclusion}

In patients undergoing laparoscopic cholecystectomy with general anesthesia, ondansetron, and aprepitant can be used to reduce postoperative nausea and vomiting, while the effect of aprepitant on this complication is greater than ondansetron. If these two drugs are used concurrently, they will have a longer and much more efficacy than their separate injections.

\section{ACKnOWLedgments}

We would like to thank all personnel in the general operating room and surgical ward for their contribution to this study.

\section{Disclosure}

\section{Ethical Approval Statement}

All procedures performed in the study involving human participants were in accordance with the ethical standards of the institutional and/or national research committee and with the 1964 Helsinki declaration and its later amendments or comparable ethical standards.

\section{Informed Consent Statement}

Informed consent was obtained from the participants included in the study.

\section{References}

1. Rahman MH, Beattie J. Post-operative nausea and vomiting. Pharmac J 2004;273:786-788.

2. Gan TJ. Risk factors for postoperative nausea and vomiting. Anesth Analg 2006;102(6):1884-1898. DOI: 10.1213/01.ANE.0000219597. 16143.4D.
3. Akhoundzadeh R, Mohtadi AR, Nesioonpour S, et al. Evaluation of the effect of injection of bupivacaine in reducing nausea and vomiting after laparoscopic cholecystectomy surgery. Jundishapur Sci Med J 2013;12(3):307-312.

4. Jafari M, Molavi B, Shojaiefard A, et al. Postoperative pain relief following bupivacaine injection in sites \& intraperitoneal spray of laparoscopic cholecystectomy. J Pharm Res Int 2017;19(4):1-7. DOI: $10.9734 / J P R I / 2017 / 36222$.

5. Miller RD. Miller's Anesthesia, 6th ed. Churchill, Livingstone: Elsevier; 2005. p. 68, 71. Ku CM, Ong BC.

6. SahabiSM, Al Reza H, AlijanpourE, etal.Comparison of dexamethasone and ondansetron with dexamethasone and metoclopramide in prevention of postoperative nausea and vomiting. Medical J of MUMS 2009;53(2): 73-78.

7. Sinha AC, Singh PM, Williams NW, et al. Aprepitant's prophylactic efficacy in decreasing postoperative nausea and vomiting in morbidly obese patients undergoing bariatric surgery. Obes Surg 2014;24(2):225-231. DOI: 10.1007/s11695-013-1065-1.

8. Apfel CC, Malhotra A, Leslie JB. The role of neurokinin-1 receptor antagonists for the management of postoperative nausea and vomiting. Curr Opin Anaesthesiol 2008;21(4):427-432. DOI: 10.1097/ ACO.0b013e328301831c.

9. Jamshid S, Mousavi A, Mohammad Reza S, et al. The effect of dexamethasone on the reduction of nausea and vomiting after thymppanomastoidectomy. J Tehran Univ Med Sci 2014;16(1): 9-13.

10. EntezariasI M, Isazadehfar K, Noorani Z. The comparison effect of preoperative ondansetron and metoclopramide in reducing nausea and vomiting after laparoscopic cholecystectomy. J Ardabil Univ Med Sci 2013;13(2):155-163.

11. Akhoundzadeh R, Mohtadi A, Nesioonpour S, et al. Evaluation of the effect of injection of bupivacaine in reducing nausea and vomiting after laparoscopic cholecystectomy surgery. J Shiraz Univ Med Sci 2016;12(3):10-14.

12. Mortazavi Y, Nikbakhsh N, Alijanpour E, et al. Effect of metoclopramide and ondansetrone plus dexamethason on postoperative nausea and vomiting in cholecystectomy laparoscopic surgery. J Gorgan Univ Med Sci 2014;16(1):9-13.

13. Isa Zadeh Far, Memory, Expectant, Fahim. Comparison of the effect of preoperative ondansetron and dexamethasone on the prevention of nausea and vomiting after laparoscopic cholecystectomy. J Mazandaran Univ Med Sci;25(133):277-284.

14. Lee SJ, Lee SM, Kim SI, et al. The effect of aprepitant for the prevention of postoperative nausea and vomiting in patients undergoing gynecologic surgery with intravenous patient controlled analgesia using fentanyl: aprepitant plus ramosetron vs ramosetron alone. Korean J Anesthesiol 2012;63(3):221-226. DOI: 10.4097/ kjae.2012.63.3.221.

15. Green MS, Green P, Malayaman SN, et al. Randomized, double-blind comparison of oral aprepitant alone compared with aprepitant and transdermal scopolamine for prevention of postoperative nausea and vomiting. Br J Anaesth 2012;109(5):716-722. DOI: 10.1093/bja/ aes233.

16. Diemunsch P, Gan TJ, Philip BK, et al. Single-dose aprepitant vs ondansetron for the prevention of postoperative nausea and vomiting: a randomized, double-blind phase III trial in patients undergoing open abdominal surgery. Br J Anaesth 2007;99(2): 202-211. DOI: 10.1093/bja/aem133.

17. Vallejo MCAL, Ibinson JW, Barnes LR, et al. Aprepitant plus ondansetron compared with ondansetron alone in reducing postoperative nausea and vomiting in ambulatory patients undergoing plastic surgery. Plast Reconstr Surg 2012;129(2):519-526. DOI: 10.1097/PRS.0b013e31822b6932. 